\title{
Clinical Application of a Complex of Blood Pressure Profile, Arterial Stiffness and Albuminuria for Cardiorenal Risk Assessment in Diabetic Patients
}

\author{
Alireza Khoshdel ${ }^{*}, 1,2$, Kerry Bowen ${ }^{2}$, Shane Carney $^{2}$, Geoffrey Eather ${ }^{2}$, John Fowler ${ }^{2}$, \\ Alastair Gillies ${ }^{2}$ and Kathryn James ${ }^{2}$ \\ ${ }^{I}$ Faculty of Health, AJA University of Medical Sciences, Tehran, Iran \\ ${ }^{2} J o h n$ Hunter Hospital, The University of Newcastle, Callaghan, Newcastle, NSW, Australia
}

\begin{abstract}
Background: In modern epidemiology, risk assessment is a crucial step in diabetes care. Clinic blood pressure reading though is not a good measurement for this purpose since both uncontrolled hypertension and white coat hypertension $(\mathrm{WCH})$ are frequent among patients with diabetes mellitus (DM). Given the problems with clinical application of ambulatory blood pressure monitoring (ABPM), in this study we evaluated clinical utility of home self measurement (HSM) with a wrist-cuff device in DM patients with hypertension to make a BP profile. Also, the clinical application of a complex of arterial study, albuminuria and blood pressure profile, in DM risk assessment was investigated. Methods and Materials: Seventy-eight adult DM patients with labile or uncontrolled hypertension were randomly assigned to 24 hour ABPM or HSM for 4 consecutive days and their BP profiles were evaluated in conjunction with an assessment of arterial stiffness and renal function as well as lipid profile. Results: The two groups were of comparable age, gender, BP, DM duration and control, smoking, lipids, renal function, arterial compliance and antihypertensive medication use. ABPM detected 33\% WCH and 17.6\% evening/night-time dipping, compared to $32 \%$ and $16 \%$ respectively for HSM, with overlapping $95 \%$ confidence intervals for day versus night BP regression coefficients. WCH patients had more compliant arteries as well as less albuminuria compared to the sustained hypertensive group. Conclusion: A complex of BP profile (by either ABPM or HSM), arterial compliance and albuminuria is a reliable and economical alternative to current methods for risk assessment in hypertensive diabetic patients.
\end{abstract}

Keywords: Blood pressure monitoring, diabetes, arterial compliance, microalbuminuria.

\section{INTRODUCTION}

Hypertension is common in Diabetes Mellitus (DM) with $71-93 \%$ of diabetic patients being hypertensive [1] . Unfortunately blood pressure (BP) control is more difficult in DM. A large study reported that while more than $63 \%$ of DM patients needed more than one antihypertensive medication, BP control was achieved in only $18 \%$, markedly lower than the non-DM group [2]. Hypertension detection and control is therefore of paramount importance in this population and yet clinic BP measurement is often unreliable with a substantial number of patients having WCH and probably misclassified [3] as poorly controlled. More importantly, the pattern of BP fluctuations can lead to a better risk assessment of patients with diabetes, chronic kidney disease and cardiovascular diseases. For instance, non-dipping nocturnal BP is now established as a CV early risk marker and associated with target organ damage, even in healthy normotensive population $[4$, 5].

Ambulatory blood pressure monitoring (ABPM) is the gold standard for $\mathrm{WCH}$ diagnosis $[3,6]$. Furthermore, it

*Address correspondence to this author at the Department of Epidemiology, Faculty of Medicine, AJA University of Medical Sciences, P.O. Box 16315-

781, Tehran, Iran; Tel/Fax: +9821-88337909;

E-mail: alikhoshdel@yahoo.com measures night-time $\mathrm{BP}$, illustrates $\mathrm{BP}$ and heart rate variability and can indirectly assesses arterial compliance [7,8]. Consequently, it is superior to clinic BP in predicting cardiovascular events and all-cause mortality [9-11]. Moreover, in PAMELA study it had a better prognostic value for all-cause mortality when compared to other methods of BP measurements in a general population over 11 years of follow-up. However, ABPM is frequently accompanied by patient discomfort and intolerance, particularly if repeated, and because of its expense it is not widely available in clinical practice. Instead, home-self measurement (HSM) is now a popular alternative being more convenient, significantly cheaper and also suitable for patient follow-up [12]. It is also a more robust predictor of cardiovascular and all-cause mortality than clinic BP [10]. While arm-cuff devices have usually been used for HSM, wrist-cuff devices are now commonly used by patients because of ease of use, particularly in the elderly, the handicapped and those with large upper arms [13]. However, despite these advantages, most wrist-cuff devices are not recommended by the British and European societies of hypertension [14, 15] and the issue of arm position is still debated $[16,17]$.

The aim of this study was firstly evaluating BP profile by either ABPM (with an automated device) or HSM (using a validated wrist-cuff monitor) for risk assessment in patients 
with DM to investigate the potential substitution of simple measurement for a complicated procedure. In addition, association between aortic pulse wave velocity and pulse wave analysis as surrogated of arterial stiffness, albuminuria and white coat condition was investigated to evaluate their potential clinical application for the risk assessment in DM subjects in conjunction with BP profile.

\section{MATERIALS AND METHODS}

\section{Subjects}

Ninety consecutive outpatient adult DM patients with labile or uncontrolled hypertension entered to the study. They were randomly assigned to 24 hour ABPM or HSM for 4 consecutive days (60 and 30 persons respectively) and their BP profiles were evaluated in conjunction with an assessment of arterial stiffness and renal function as well as lipid profile. However, 78 patients $(86.6 \%)$ complied with the instructions including 52 and 26 individuals in ABPM and HSM groups respectively.

The study subjects consisted of $\mathbf{7 8}$ adult DM patients with labile or uncontrolled BP attending an outpatient diabetic clinic. Patients with atrial fibrillation, severe heart disease, renal transplant, end-stage renal disease, severe renovascular disease, primary hyperaldostronism, pheochromocytoma, and pregnant women were excluded. Each participant provided informed consent for the study, which was approved by the Human Research Ethics Committee, The University of Newcastle and the Hunter-New England Health Ethics Committee (NSW, Australia). In this selected DM population, $82 \%$ had type 2 DM, mean age was $61 \mathrm{y}$., $51 \%$ were male and $13 \%$ smokers (Table 1). Twenty-five

Table 1. Patients' Characteristics in the BP Monitoring Groups, all P Values $>0.05$

\begin{tabular}{|c|c|c|c|}
\hline & $\begin{array}{c}\text { ABPM } \\
n=52\end{array}$ & $\begin{array}{l}\text { HSM } \\
n=26\end{array}$ & P-value \\
\hline Age $(y)$ & $61.7(1.5)$ & $60.6(2.4)$ & 0.71 \\
\hline Male $(\%)$ & 53 & 46 & 0.81 \\
\hline Smoker $(\%)$ & 14 & 12 & 1.00 \\
\hline DM duration (y.) & $11.3(1.7)$ & $10.4(1.7)$ & 0.73 \\
\hline HbA1c $(\%)$ & $7.5(0.2)$ & $7.5(0.9)$ & 0.43 \\
\hline Cholesterol (mmol/l) & $4.7(0.1)$ & $4.4(0.2)$ & 0.17 \\
\hline Triglyceride (mmol/l) & $1.8(0.1)$ & $1.8(0.1)$ & 0.21 \\
\hline eGFR (MDRD)ml/min/1.73 m2 & $74.6(3.3)$ & $77.4(4.2)$ & 0.61 \\
\hline Clinic SBP (mmHg) & $148.0(2.6)$ & $148.0(3.4)$ & 0.95 \\
\hline Clinic DBP (mmHg) & $82.9(1.8)$ & $79.6(2.1)$ & 0.27 \\
\hline Clinic Heart Rate (bpm) & $70.9(2.3)$ & $71.9(2.5)$ & 0.78 \\
\hline 5 year estimated CV risk $(\%)$ & $45(2)$ & $42(2)$ & 0.44 \\
\hline Adjusted Alx (\%) & $22.7(1.7)$ & $24.2(0.7)$ & 0.53 \\
\hline CF-PWV (m/s) & $12.2(0.4)$ & $12.4(0.7)$ & 0.78 \\
\hline Average number of anti-HT meds & $2.3(0.2)$ & $2.2(0.3)$ & 0.70 \\
\hline
\end{tabular}

percent had a history of ischemic heart disease, $13 \%$ a previous cerebrovascular event, $8 \%$ peripheral vascular disease and $72 \%$ were hyperlipidemic or taking lipid lowering medication. Sixty four percent were taking more than 1 medication for BP, which included $47 \%$ angiotensin receptor blockers, 46\% ACE inhibitors, 43\% diuretics, 39\% beta blockers, 38\% calcium channel blockers, 10\% alpha-blockers and $4 \%$ vasodilators. In respect to diabetes medication, $47 \%$ of the individuals were taking a biguanide, $15 \%$ a sulphonylurea, 4\% from other oral hypoglycemic agents; and $53 \%$ on insulin. Forty two percent were taking aspirin, 11\% occasionally used non-steroidal anti-inflammatory drugs and $4 \%$ were on anti-coagulant agents.

\section{Measurements}

Participants were randomly allocated to an automated 24 hour ABPM (Spacelabs 90219) or 4 consecutive days of a validated oscillometric wrist-cuff HSM device (Omron HEM-609). Two-third of subjects was allocated to ABPM (i.e. 52 ABPM and $26 \mathrm{HSM}$ ) since it is currently the preferred option by clinical practice guidelines from related authorities [3, 18-21]. BP was measured every 20 minutes during the day and every hour at night with ABPM. HSM included five measurements at approximately 0800, 1200, 1600,1800 and before going to bed, during each of the 4 consecutive days and the recorded patients' logs were compared to the devices inbuilt memory for measurement validation. All patients were informed about monitor use including correct arm position and were provided with instructions.

In order to calibrate HSM (group A) with ABPM (group B), the day time was assumed to be from 0600 to 1800 and evening/night time from 1800 to $0600 \mathrm{hrs}$. The criteria for $\mathrm{WCH}$ diagnosis was ambulatory average daytime $\mathrm{BP} \leq 135$ / $85 \mathrm{mmHg}$ despite a hypertensive state as measured in clinic or office $[3,20]$. Although HSM did not include sleeping $\mathrm{BP}$, the average of two evening BP measurements in the period of monitoring was used as a surrogate for nighttime BP in the group A, because European Society of Hypertension clearly suggested that nocturnal hypertension may be suspected on the basis of increased evening home blood pressure [3]. Nocturnal dipping was calculated as [(mean daytime $\mathrm{BP}$ - mean nighttime $\mathrm{BP}$ )/ mean daytime $\mathrm{BP}]$ and patients with less than 10\% SBP dipping were identified as non-dippers $[3,22]$. Also the absolute white coat effect and nighttime BP reduction were considered in the analysis. As stated in PAMELA study the day to night time ratio was applied as a marker of BP pattern [10] .

Arterial compliance was measured by pulse wave velocity (Complior ${ }^{\circledR}$, Colson, Paris, France) and Pulse Wave analysis (SphygmoCor ${ }^{\circledR}$, AtCor Medical, Sydney, New South Wales, Australia) and pulse pressure was considered as an indirect measure [23]. Pulse wave velocity (PWV) measurement involved the placement of sensors over the carotid and femoral arteries, the carotid-femoral (CF) velocity being an index of central arterial stiffness [24]. Pulse wave analysis was measured using a sensor on the radial artery, resulting in the measurements of augmentation index (AIx), adjusted for heart rate, ejection duration (ED), the time of the reflected wave (rTr) as well as peripheral and aortic pulse pressure (PP). Estimation of cardiovascular risk 
was based on 11 risk factors to quantify an adult's risk of death from cardiovascular disease, including stroke and coronary heart disease, as calculated by the "Risk Score Calculator" (www.riskscore.org.uk). Kidney function was assessed by MDRD formula (eGFR) (http://nephron.com/ cgi-bin/MDRD_NKF.cgi).

\section{Analysis}

For validation purposes, ABPM was considered as the gold standard. The ABPM group was double the HSM group in sample size which increased the power of the study analyses. The two groups were compared by t-test for normally distributed variables and Mann-Whitney U test were applied for non-normally distributed ones. The proportion of the nominal variables was compared by $\mathrm{Chi}^{2}$ between the two groups. Regression coefficient of the day versus night natural logarithm of BP (as an index for BP night-dipping) was calculated with $95 \%$ confidence interval (CI), separately for SBP and DBP and for both BP monitoring methods in order to know whether this association is consistent and comparable between the two methods of BP monitoring. The power of tests for desired detectable level for BP, as much as 2 to 5 $\mathrm{mmHg}$ was 91 to $99 \%$ and also $85 \%$ for detection of $0.5 \mathrm{~m} / \mathrm{s}$ difference in PWV. P value was considered significant if it was less than 0.05 for all tests. Correlation of the continuous variables was carried out by Spearman method. SPSS-12 software was applied for analyses.

\section{RESULTS}

\section{BP Profile}

The HSM and ABPM groups were comparable regarding their age, gender, DM duration, clinic BP, arterial compliance, glycemic control, renal function and lipid profile as well as estimated 5-year cardiovascular risk score (Table 1). There was no significant difference in the medication use between the two groups except insulin use which was more frequent in the HSM group (79\% compared to $42 \%$ ).

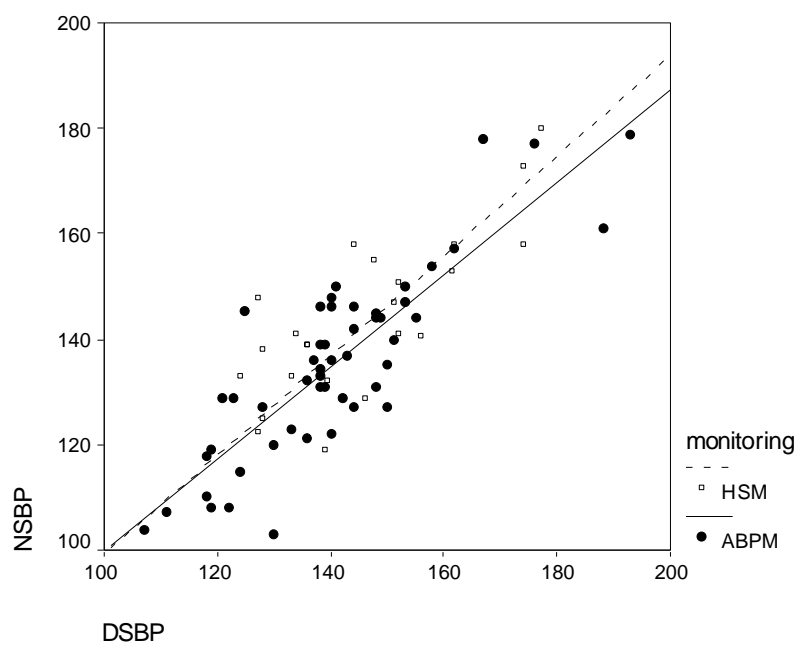

The BP monitoring results demonstrated that $33 \%$ of all subjects had WCH, 83\% were non-dippers for SBP and $71 \%$ non-dippers for DBP. Interestingly, the recorded mean SBP and DBP as well as the daytime and evening/nighttime SBP and DBP by HSM and ABPM were comparable. Moreover, there was no significant difference between the two methods in detecting $\mathrm{WCH}$ and the non-dipper conditions $(\mathrm{P}>0.05)$ (Table 2).

Table 2. BP Monitoring Results

\begin{tabular}{|c|c|c|c|}
\hline & ABPM & HSM & $\begin{array}{c}\mathbf{P} \\
\text { value }\end{array}$ \\
\hline \multirow[t]{2}{*}{ 24-hr } & $138.6(2.4)$ & $143.6(3.1)$ & 0.21 \\
\hline & $77.1(1.6)$ & $78.9(3.0)$ & 0.57 \\
\hline \multirow[t]{2}{*}{ Day-time (0600-1800) } & $140.6(2.4)$ & $144.1(3.3)$ & 0.39 \\
\hline & $79.1(1.6)$ & $79.5(3.1)$ & 0.90 \\
\hline \multirow[t]{2}{*}{ Night/ Evening -time } & $135.4(2.5)$ & $140.9(4.0)$ & 0.23 \\
\hline & $72.8(1.6)$ & $76.5(2.8)$ & 0.22 \\
\hline Night/Evening Dippers (\%) & 17.9 & 16.0 & 0.27 \\
\hline $\begin{array}{c}\text { White-Coat Hypertension diagnosis } \\
(\%)\end{array}$ & 33 & 32 & 1.00 \\
\hline
\end{tabular}

When log day BP was regressed against log evening/ night BP (separately for SBP and DBP), 95\% CIs of the regression coefficient (slop $\beta$ ) were widely overlapping and did not show any difference between HSM and ABPM (Table 3, Fig. 1), though SBP in higher levels and DBP in lower levels were slightly deviated from ABPM.

Of interest, smokers were more likely to be a dipper $(\mathrm{OR}=7.2,95 \% \mathrm{CI}=1.7$ to 30.7$)$.

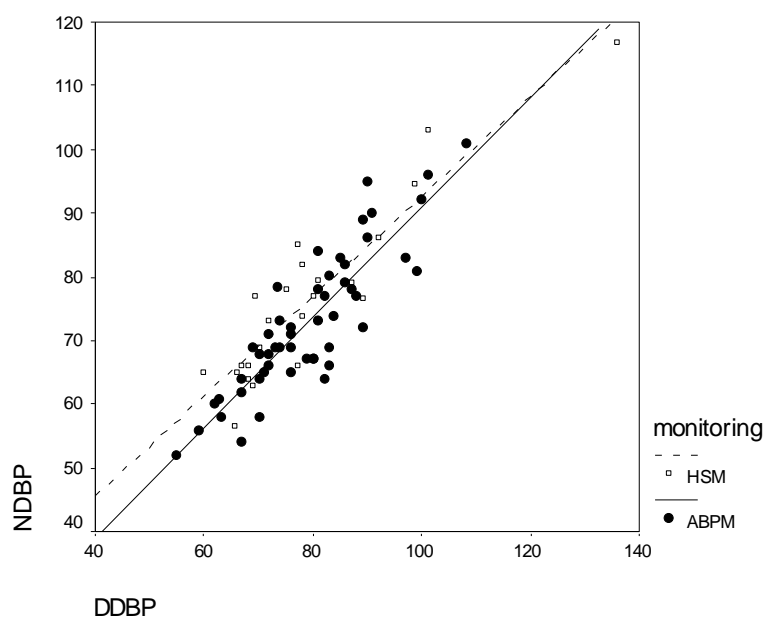

Fig. (1). Day (D) against night $(\mathrm{N})$ blood pressure $(\mathrm{mmHg})$ for systolic (SBP) (left) and diastolic (DBP) (right) measurements by ambulatory blood pressure monitoring (ABPM-solid line) and home self-measurement (HSM- dashed line). The figure demo nstrates that, HSM overstimates NSBP in high levels of SBP, however the difference is minimal when compared to ABPM. For DBP, disagreement is more prominent in lower levels of BP, with HSM overstimating NDBP. However the difference is minimal in usual levels of BP. 
Table 3. Comparison of the Log Regression Slop (with $95 \%$ CI) of the Day against Night Blood Pressure for Systolic and Diastolic Readings

\begin{tabular}{|c|c|c|}
\hline & ABPM & HSM \\
\hline \hline Log Day-Night SBP & & \\
Constant & $0.14(-0.2$ to 0.5$)$ & $-0.15(-1.1$ to 0.8$)$ \\
B (slope) & $0.92(0.76$ to 1.09$)$ & $1.06(0.62$ to 1.50$)$ \\
\hline Log Day-Night DBP & & \\
Constant & $1.36(0.92$ to 1.80$)$ & $0.28(-0.06$ to 0.62$)$ \\
B (slope) & $0.40(0.17$ to 0.64$)$ & $0.84(0.66$ to 1.02$)$ \\
\hline
\end{tabular}

\section{White Coat Hypertension State}

Comparison of the sustained or ambulatory hypertensive $(\mathrm{AH})$ and $\mathrm{WCH}$ patients revealed that DM duration, glycemic control, cholesterol and eGFR were not significantly different between these two categories. In contrast, CF-PWV was significantly lower in WCH subjects $(\mathrm{P}=0.03$ by t-test and $\mathrm{p}=0.01$ by Mann-Whitney $U$ ), despite Alx being comparable between the two groups. Likewise no statistical significant difference was detected between the two groups regarding ED and rTr. While peripheral and aortic PP were comparable between the two groups, ambulatory day and night time PPs were significantly lower for $\mathrm{WCH}$ than within the sustained hypertensive group $(\mathrm{P}<0.0001)$. In addition, patients with $\mathrm{WCH}$ had less albuminuria $(\mathrm{P}=0.02)$ (Table 4, Fig. 2).

No relationship was detected between $\mathrm{WCH}$ or nondipper conditions and DM duration, gender, history of cardiovascular disease and hyperlipidemia.

\section{DISCUSSION}

In this study approximately $32 \%$ of DM clinic patients with poorly controlled hypertension diagnosed at a diabetic clinic had WCH and only $17 \%$ of the patients had a significant evening/night dipping SBP. Of particular importance a four day HSM with 3 daytime and 2 evening time readings was practically as useful as 24 hour ABPM in detecting $\mathrm{WCH}$ and non-dipper conditions. It was also revealed that patients with $\mathrm{WCH}$ had better arterial compliance and less albuminuria than those with sustained hypertension.

The utility of HSM as an alternative to ABPM in the detection of WCH has long been an issue. However Stergiou et al have shown that HSM can be used for WCH diagnosis [25] and it has recently been accepted for screening of $\mathrm{WCH}$, although the diagnosis should be confirmed by ABPM [26, 27]. Nevertheless, BP measurement guidelines from the British and the European hypertension societies [15, 28, 29] do not support using devices that measure BP in the finger or in the arm below the elbow until they are validated according to international protocols. Therefore, we applied a validated device for this purpose. However the number of reports demonstrating the validity of wrist-cuff monitors is increasing [30-35], though with particular considerations including wrist position[16]. Furthermore, in a recent study a wrist monitor was comparable to an automated upper arm device and closer to the mercury sphygmomanometer [32] and in another, the result of the wrist monitor was closer to the intra-arterial BP than a mercury device [30]. In this study we used a popular wrist-cuff BP monitor $[17,35]$ which has been validated and described by patients as convenient and easy to use. In order to compare non-dipping detection with HSM and ABPM, night-time blood pressure was considered to be after 1800, and while this is not the usual ABPM time, a similar time has been applied during another DM study

Table 4. Vascular Indices for Patients with Sustained Hypertension and White-Coat Hypertension

\begin{tabular}{|c|c|c|c|}
\hline & $\begin{array}{c}\mathbf{A H} \\
\mathbf{N}=52\end{array}$ & $\begin{array}{l}\text { WCH } \\
\mathbf{N}=26\end{array}$ & P Value \\
\hline Diabetes duration (months) & $120(36-201)$ & $84(5-156)$ & 0.18 \\
\hline HbAlc $(\%)$ & $7.4(6.6-8.4)$ & $7.3(6.6-8.2)$ & 0.98 \\
\hline Cholesterol (mmol/l) & $4.7(3.9-5.1)$ & $4.6(3.8-5.1)$ & 0.65 \\
\hline eGFR (MDRD) ml/min $/ 1.73 \mathrm{~m} 2$ & $73.6(63.3-88.8)$ & $76.6(56.6-88.2)$ & 0.87 \\
\hline Albumin Creatinine Ratio (mg/mmol) & $1.8(1.1-3.5)$ & $0.7(0.3-2.4) \#$ & 0.02 \\
\hline Adjusted Alx (\%) & $25.0(17.5-30.5)$ & $24.0(19.2-29.0)$ & 0.92 \\
\hline CF-PWV (m/s) & $12.4(11.1-14.1)$ & $10.2(9.3-12.3) *$ & 0.01 \\
\hline Peripheral PP (mmHg) & $68.0(56.7-82.0)$ & $59.5(48.0-75.0)$ & 0.11 \\
\hline Aortic PP (mmHg) & $51.0(38.0-69.0)$ & $48.5(35.0-54.7)$ & 0.29 \\
\hline Ambulatory Day pulse pressure & $68.0(62.5-74.0)$ & $53.0(46.0-59.0) \S$ & 0.0001 \\
\hline Ambulatory Night PP & $69.0(62.0-76.0)$ & $56.0(43.2-61.7) \S$ & 0.0001 \\
\hline Non-dipper SBP (\%) & $78.8 \%$ & $80.2 \%$ & 0.87 \\
\hline $\mathrm{ED}(\%)$ & $35(31-38)$ & $33.5(31.0-38.5)$ & 0.55 \\
\hline $\mathrm{rTr}(\mathrm{ms})$ & $0.154(0.135-0.185)$ & $0.151(0.133-0.178)$ & 0.64 \\
\hline
\end{tabular}

Values are median (inter-quartile range) unless specified; Alx: augmentation index, CF: carotid-femoral, PWV: pulse wave velcity, ED: Ejection duration, rTr: relative time for reflected wave return

\# $\mathrm{P}=0.02, * \mathrm{P}=0.01, \S \mathrm{P}=0.0001$; all non parametric tests 

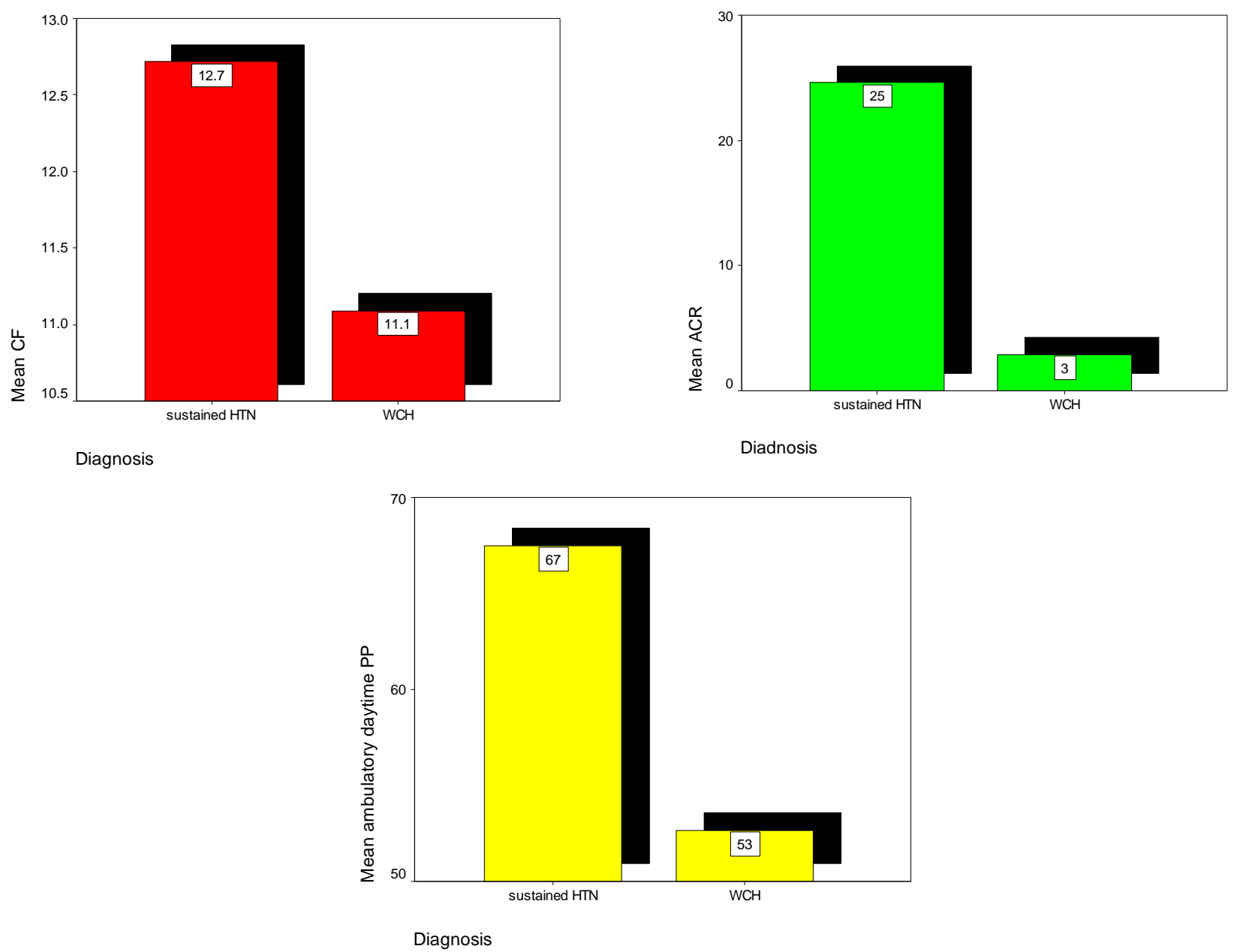

Fig. (2). Mean central arterial stiffness (CF-PWV), albumin to creatinine ratio (ACR) and ambulatory daytime pulse pressure in ambulatory or sustained hypertensive and white-coat hypertensive groups.

[36]. In that study, Masding et al. compared average BP readings at 1300 and 2300 by HSM over 3 days in 55 diabetic patients and considered a BP fall of $20 / 10 \mathrm{mmHg}$ as a criterion for night-dippers. They concluded that this approach despite its sensitivity, was not specific enough to detect the non-dipping condition, although was far superior to clinic BP in the detection of true hypertension. Using a wrist-cuff monitor and different criteria for night-dipping diagnosis, our results suggest that the average evening BP reduction measured by HSM, may predict BP dipping during sleep.

Previous reports on the frequency of $\mathrm{WCH}$ in DM widely vary from 23 to $62 \%$ for type 2 and 68 to $75 \%$ for type $1 \mathrm{DM}$ [37-42]. However, this variation is partially due to definition differences. For instance Burgess et al., who reported a prevalence of $62 \% \mathrm{WCH}$ in DM, identified $\mathrm{WCH}$ as mean day $\mathrm{BP}<$ mean clinic BP. Applying this description in our study would result in a $71 \%$ prevalence of $\mathrm{WCH}$.

The relationship between ABPM findings and diabetes complications including nephropathy has scarcely been reported [37, 43-45]. Nielsen et al. also reported $23 \% \mathrm{WCH}$ in diabetic patients with normoalbuminuria, $8 \%$ in microalbuminuria and $9 \%$ in nephropathy [38], which is in agreement with our findings. The greater likelihood of albuminuria in sustained hypertensive patients when compared to $\mathrm{WCH}$ reflects the severity of general vascular damage in this population and is consistent with the increased CF-PWV in the sustained hypertensive group [46]. While reduced CFPWV and therefore arterial stiffness noted in DM patients with WCH has not been previously reported, it is consistent with a report in non-DM patients [47]. Given PP as an indirect measure of arterial stiffness, it was interesting that neither peripheral nor aortic PP was different between sustained hypertensives and $\mathrm{WCH}$, but ambulatory day and night PP were significantly lower in $\mathrm{WCH}$. This is in line with some other reports of a greater predicitivity of ambulatory obtained BP profile for mortality risk [48].

The above evidence suggests that in the presence of albuminuria and increased arterial stiffness, $\mathrm{WCH}$ would be less likely and therefore clinic BP is more likely to represent the real BP. Also, PP is not a valid marker of arterial stiffness unless the PP estimation is based on ambulatory BP measurement.

While $83 \%$ of our study population were non-dippers, compared with 30 to $64 \%$ in other studies $[42,45,49]$, the greater frequency of non-dipper in our study is probably 
related to our selection criteria, since only patients with labile or uncontrolled hypertension were selected. We did not find any relationship between non-dipping condition and DM duration, HbA1c and microalbuminuria, which supports previous studies. Nevertheless, non-dipping is now a proven risk for all-cause and cardiovascular mortality $[4,50]$ and vascular events [45] in DM. Considering that the short-term reproducibility of the non-dipping pattern in DM patients is better than in non-DM hypertensive patient [49], BP monitoring may provide an opportunity for a better risk assessment in DM patients. However, consideration of a cut-point rather than using a continuous value in the BP dipping definition is criticized by some authors [22].

Finally, the current study, enhances the previous evidence of the role of arterial assessment in risk assessment and better management of CVD, particularly in DM [51].

In conclusion, HSM using a validated wrist-cuff monitor is as useful as ABPM in identifying DM patients with $\mathrm{WCH}$ in this population. Furthermore, HSM can be used to detect nocturnal non-dipping BP in such individuals. Of particular interest, it was observed that DM patients with $\mathrm{WCH}$ manifest more compliant arteries and less albuminuria when compared to DM individuals with sustained hypertension. This provides a battery for easy renal-cardiac risk assessment in $\mathrm{DM}$ population.

\section{ACKNOWLEDGEMENT}

The Authors would like to acknowledge the support of Dr. J. Lowe, Dr. T. Donnelly, Dr. S. Linjawi and Dr. E. Tyson as well as Hunter New England Health Services and Diabetes Education Centers.

\section{CONFLICTS OF INTEREST}

None declared.

\section{Appendix}

\section{Summary}

\begin{tabular}{|l}
\hline 'What is known about topic' \\
- White-coat hypertension (WCH) is a common finding in \\
diabetes mellitus \\
While automatic ambulatory blood pressure monitoring \\
(ABPM) is the gold standard for the WCH, it is often not very \\
convenient, unrepeatable, expensive and unavailable in practice \\
- Home self BP measurement (HSM), in particular with popular \\
wrist-cuff devices, is more convenient, repeatable and cheaper, \\
but must be validated in the target groups. \\
-What this study adds' \\
HSM with a wrist-cuff device had comparable BP profile result \\
with ABPM in two comparable groups with diabetes and \\
hypertension \\
Average evening BP measured by HSM may predict BP dipping \\
during night \\
Patients with WCH had less central arterial stiffness (as \\
measured by CF-PWV and ambulatory PP) and albuminuria \\
than the sustained hypertensive group. Therefore these markers \\
may predict the likelihood of WCH in clinical practice
\end{tabular}

\section{REFERENCES}

[1] Tarnow L, Rossing P, Gall MA, et al. Prevalence of arterial hypertension in diabetic patients before and after the JNC-V. Diabetes Care 1994; 17(11): 1247-51.

[2] Obara T, Ohkubo T, Kikuya M, et al. The current status of home and office blood pressure control among hypertensive patients with diabetes mellitus: the Japan home versus office blood pressure measurement evaluation (J-HOME) study. Diabetes Res Clin Pract 2006; 73(3): 276-83

[3] O'Brien E, Asmar R, Beilin L, et al. European society of hypertension recommendations for conventional, ambulatory and home blood pressure measurement. J Hypertens 2003; 21(5): 82148.

[4] Ohkubo T, Hozawa A, Yamaguchi J, et al. Prognostic significance of the nocturnal decline in blood pressure in individuals with and without high 24-h blood pressure: the Ohasama study. J Hypertens 2002; 20 (11): 2183-9.

[5] Hoshide S, Kario K, Hoshide Y, et al. Associations between nondipping of nocturnal blood pressure decrease and cardiovascular target organ damage in strictly selected communitydwelling normotensives. Am J Hypertens 2003; 16(6): 434-8.

[6] McGrath BP. Ambulatory blood pressure monitoring. Med J Aust 2002; 176(12): 588-92.

[7] Singh RB, Cornelissen G, Weydahl A, et al. Circadian heart rate and blood pressure variability considered for research and patient care. Int J Cardiol 2003; 87(1): 9-28; discussion 29-30.

[8] Kikuya M, Hozawa A, Ohokubo T, et al. Prognostic significance of blood pressure and heart rate variabilities: the Ohasama study. Hypertens J 2000; 36(5): 901-6.

[9] Hansen TW, Jeppesen J, Rasmussen S, et al. Ambulatory blood pressure and mortality: a population-based study. Hypertens J 2005; 45(4): 499-504.

[10] Sega R, Facchetti R, Bombelli M, et al. Prognostic value of ambulatory and home blood pressures compared with office blood pressure in the general population: follow-up results from the Pressioni Arteriose Monitorate e Loro Associazioni (PAMELA) study. Circulation 2005; 111(14): 1777-83.

[11] Dolan E, Stanton A, Thijs L, et al. Superiority of ambulatory over clinic blood pressure measurement in predicting mortality: the dublin outcome study. Hypertension 2005; 46(1): 156-61.

[12] Cuspidi C, Meani S, Salerno M, et al. Reproducibility of nocturnal blood pressure fall in early phases of untreated essential hypertension: a prospective observational study. J Hum Hypertens 2004; 18(7): 503-9.

[13] de Lusingnan S, Thiru K, Meredith K, et al. Measuring blood pressure at the wrist: more comfortable for patients and more convenient for doctors. Public Health 2000; 114(3): 165-8.

[14] O'Brien E, Petrie JC, Littler WA, et al. Blood pressure measurement: recommendations from british hypertension Society, London. in BHS booklets. 1999, Available at: www.abdn.ac.uk/medical/ bhs/booklet.

[15] O'Brien E, Waeber B, Parati G, et al. Blood pressure measuring devices: recommendations of the european society of hypertension BMJ 2001; 322(7285): 531-6.

[16] Khoshdel AR, Carney S, Gillies A. The impact of arm position and pulse pressure on the validation of a wrist-cuff blood pressure measurement device in a high risk population. Int J Gen Med 2010; 2010(3): 119-25.

[17] Khoshdel AR, Carney S. Wrist-cuff blood pressure home selfmeasurement in diabetic patients: comparable to ambulatory blood pressure monitoring. Hypertension 2007; 49(6): 1470.

[18] O'Brien E, Pickering T, Asmar RG, et al. Working group on blood pressure monitoring of the europian society of hypertension intertational protocol for validation of blood pressure measuring devices in adults. Blood Press Monit 2002; 7: 3-17.

[19] O'Brien E, Waeber B, Parati G, et al. Blood pressure measuring devices: recommendations of the europian society of hypertension. BMJ 2001; 322: 531-5.

[20] O'Brien E, Coats A, Owens P, et al. Use and interpretation of ambulatory blood pressure monitoring: recommendations of the British hypertens society. BMJ 2000; 320(7242): 1128-34.

[21] Imai $\mathrm{Y}$, Otsuka $\mathrm{K}$, Kawano $\mathrm{Y}$, et al. Japanese society of hypertension guidelines for self-monitoring of blood pressure at home. Hypertens Res 2003; 26(10): pp. 771-82. 
[22] Chaves H, Campello de Souza FM, Krieger EM. The reproducibility of dipping status: beyond the cutoff points. Blood Press Monit 2005; 10(4): 201-5.

[23] Khoshdel AR. Time to end a doubt: is pulse wave analysis a valid measure for central arterial blood pressure and arterial stiffness? J Hypertens 2007; 25(3): 724-5.

[24] Khoshdel AR, Thakkinstian A, Carney SL, et al. Estimation of an age-specific reference interval for pulse wave velocity: a metaanalysis. J Hypertens 2006; 24(7): 1231-7.

[25] Stergiou GS, Zourbaki AS, Skeva II, et al. White coat effect detected using self-monitoring of blood pressure at home: comparison with ambulatory blood pressure. Am J Hypertens 1998; 11(7): 820-7.

[26] Stergiou G, Mengden T, Padfield PL, et al. Self monitoring of blood pressure at home. BMJ, 2004; 329(7471): 870-1.

[27] Verdecchia $\mathrm{P}$, O'Brien E, Pickering $\mathrm{T}$, et al. When can the practicing physician suspect white coat hypertension? statement from the working group on blood pressure monitoring of the European society of hypertension. Am J Hypertens 2003; 16(1): 87-91.

[28] O'Brien E, Pickering T, Asmar R, et al. Working group on blood pressure monitoring of the european society of hypertension international protocol for validation of blood pressure measuring devices in adults. Blood Press Monit 2002; 7(1): 3-17.

[29] Reims H, Kjeldson SE, Mancia G. Update on Hypertension management. Eur Soc Hypertens Sci Newslet 2002; 3(12): 1-2.

[30] Watson S, Wenzel RR, di Matteo C, et al. Accuracy of a new wrist cuff oscillometric blood pressure device: comparisons with intraarterial and mercury manometer measurements. Am J Hypertens 1998; 11(12): 1469-74.

[31] Mourad A, Gillies A, Carney S. Inaccuracy of wrist-cuff oscillometric blood pressure devices: an arm position artefact? Blood Press Monit 2005; 10(2): 67-71.

[32] Cuckson AC, Moran P, Seed P, et al. Clinical evaluation of an automated oscillometric blood pressure wrist device. Blood Press Monit 2004; 9(1): 31-7.

[33] Altunkan S, Yildiz S, Azer S. Wrist blood pressure-measuring devices: a comparative study of accuracy with a standard auscultatory method using a mercury manometer. Blood Press Monit 2002; 7(5): 281-4.

[34] Caridi G, Zoccali C, Enia G. Oscillometric wrist devices: comparison with the standard mercury sphygmomanometer and estimate of the "supine" error. G Ital Nefrol 2003; 20(6): 589-91.

[35] Graves JW. A survey of validated automated home blood pressure monitors available for the internet shopper. Blood Press Monit 2005; 10(2): 103-7.

[36] Masding MG, Jones JR, Bartley E, et al. Assessment of blood pressure in patients with Type 2 diabetes: comparison between home blood pressure monitoring, clinic blood pressure measurement and 24-h ambulatory blood pressure monitoring. Diabet Med 2001; 18(6): 431-7.
[37] Flores L, Gimenez M, Esmatjes E. Prognostic significance of the white coat hypertension in patients with type 1 diabetes mellitus. Diabetes Res Clin Pract 2006; 74(1): 21-5.

[38] Nielsen FS, Gaede P, Vedel P, et al. White coat hypertension in NIDDM patients with and without incipient and overt diabetic nephropathy. Diabetes Care 1997; 20(5): 859-63.

[39] Puig JG, Ruilope LM, Ortega R. Antihypertensive treatment efficacy in type II diabetes mellitus. Dissociation between casual and 24-hour ambulatory blood pressure. Spanish Multicenter Study Group. Hypertension 1995; 26(6 Pt 2): 1093-9.

[40] Flores L, Recasens M, Gomis R, et al. White coat hypertension in type 1 diabetic patients without nephropathy. Am J Hypertens 2000; 13(5 Pt 1): 560-3.

[41] Holl RW, Pavlovic M, Heinze E, et al. Circadian blood pressure during the early course of type 1 diabetes. Analysis of 1,011 ambulatory blood pressure recordings in 354 adolescents and young adults. Diabetes Care 1999; 22(7): 1151-7.

[42] Marczewski K, Krawczyk W, Rozyc P, et al. Day/night ratio of microproteinuria and blood pressure rhythm in type II diabetes. Diabetes Res Clin Pract 1996; 33(3): 169-72.

[43] Omvik P, Gerhardsen G. The Norwegian office-, home-, and ambulatory blood pressure study (NOHA). Blood Press 2003 12(4): 211-9.

[44] Knudsen ST, Poulsen PL, Hansen KW, et al. Pulse pressure and diurnal blood pressure variation: association with micro- and macrovascular complications in type 2 diabetes. Am J Hypertens 2002; 15(3): 244-50.

[45] Nakano S, Fukuda M, Hotta F, et al. Reversed circadian blood pressure rhythm is associated with occurrences of both fatal and nonfatal vascular events in NIDDM subjects. Diabetes $1998 ; 47(9)$ : 1501-6.

[46] Benetos A, Lacolley P. From 24-hour blood pressure measurements to arterial stiffness: a valid short cut? Hypertension 2006; 47(3): 327-8.

[47] Silveira A, Mesquita A, Maldonado J, et al. White coat effect in treated and untreated patients with high office blood pressure. Relationship with pulse wave velocity and left ventricular mass index. Rev Port Cardiol 2002; 21(5): 517-30.

[48] Dawes MG, Coats AJ, Juszczak E. Daytime ambulatory systolic blood pressure is more effective at predicting mortality than clinic blood pressure. Blood Press Monit 2006; 11(3): 111-8.

[49] Cuspidi C, Meani S, Lonati L, et al. Short-term reproducibility of a non-dipping pattern in type 2 diabetic hypertensive patients. J Hypertens 2006; 24(4): 647-53.

[50] Kario K, Pickering TG, Matsuo T, et al. Stroke prognosis and abnormal nocturnal blood pressure falls in older hypertensives. Hypertension 2001; 38(4): 852-7.

[51] Khoshdel AR, Carney SL, Nair BR, et al. Better management of cardiovascular diseases by pulse wave velocity: combining clinical practice with clinical research using evidence-based medicine. Clin Med Res 2007; 5(1): 45-52.

This is an open access article licensed under the terms of the Creative Commons Attribution Non-Commercial License (http: //creativecommons.org/licenses/by$\mathrm{nc} / 3.0 /$ ), which permits unrestricted, non-commercial use, distribution and reproduction in any medium, provided the work is properly cited. 